\title{
Programmable ultraviolet light-emitting diode array with a transfer lens as light-field adjustable source
}

\author{
Jiun-Woei Huang* \\ National Taiwan University, Institute of Applied Mechanics, Taipei, Taiwan
}

\begin{abstract}
A programmable ultraviolet light-emitting diode (UVLED) array with a transfer lens forms an adjustable intensity distribution upon a mask, whether for the uniform distribution or variable distribution for compensate wavefront error introduced by the lithographic lens, so as to fabricate an aberration-free pattern on a wafer. The collimating and aligning all rays in one aperture lens with a $N \times N$ two-dimensional UVLED array is designed and fabricated by integrating with lithographic lens to form aberration-free smallest linewidth in integrated circuit wafer. Our work provides a transfer lens for the UVLED array to manipulate the intensity distribution fitted for the aligner in the soft- and near-contact mode and steppers. (C) The Authors. Published by SPIE under a Creative Commons Attribution 4.0 Unported License. Distribution or reproduction of this work in whole or in part requires full attribution of the original publication, including its DOI. [DOI: 10.1117/1.OE .60.9.091502]
\end{abstract}

Keywords: programmable ultraviolet light-emitting diode array; two-dimensional light source distribution; uniform illumination; collecting and aligning all rays in one aperture lens.

Paper 20200198SS received Feb. 20, 2020; accepted for publication Aug. 5, 2020; published online May 4, 2021.

\section{Introduction}

The lithography system primarily integrates two subsystems to make precision nanometer-scale integrated circuit in silicon wafers: one is a condenser lens to illuminate the mask, and the other is a lithographic lens to image the pattern of the mask, both are correlated. ${ }^{1}$ In an ideal condition, to fabricate the smallest linewidth in a wafer, not only requires a spherical and coma aberrationfree lithographic lens but also a condenser optics to provide the uniform distribution at the position of the mask. In common failure cases, either one is the defect lens spherical or coma aberrations by lithographic introduced, this feature will fail to fulfill the smallest line width requirement of circuit, even if the lithographic lens is perfect, the nonuniform illuminated mask still cannot produce the qualified least small width feature (LSWF). Thus, lens quality and the light distribution in the exposed position are the most important factors to produce the successful LSWF.

\subsection{Previous Method}

As an example, Fig. 1 shows the imperfect feature in the wafers, which were two different exposure fatal results by nonuniform light intensity. Figure 1 shows after fatal exposure under nonuniform source, linewidth at 2 and $5 \mu \mathrm{m}$ shown in Figs. 1(a) and 1(b), respectively.

In the past, the imperfectly printed masked wafer could be corrected by adjusting the aperture shape of illumination optics, such as dipole, quadrupole, or metal plates to form as compensate wavefront to distribute the light source intensity to form a small linewidth in tolerance, or by applying a relative numerical aperture of lens and illumination optics and the light intensity can be adjusted to the distribution of a two-dimensional (2-D) source to compensate for the imperfect feature. ${ }^{1-3}$ Another method is by putting on a coating with different local transmittance to match the light source, to correct the uneven distribution at aperture, thus to form uniform light intensity. However, because of each different circuit, each separate block plate must be changed to meet each small linewidth requirement, and the shape of the block plate must be changed every time.

In this study, a programmable ultraviolet light-emitting diode (UVLED) array with collimated optics as a transfer lens with light-field adjustable source is proposed. It not only forms

*Address all correspondence to Jiun-Woei Huang, E-mail: jwhuang12345@gmail.com 


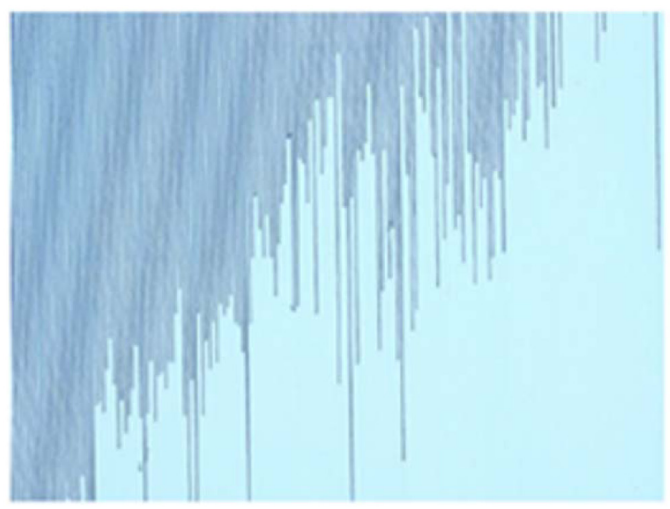

(a)

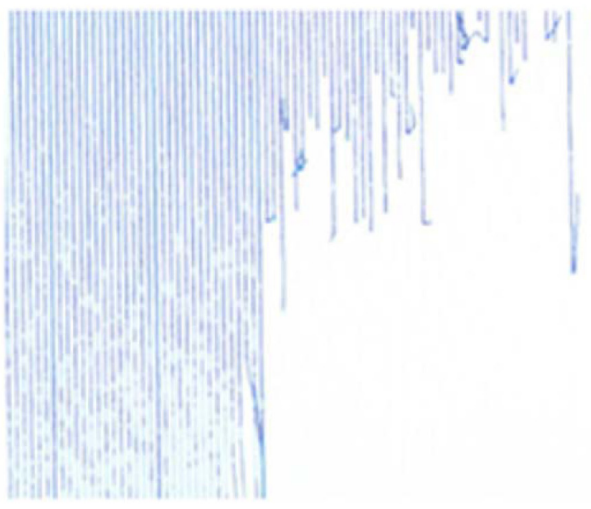

(b)

Fig. 1 Fatal exposure under nonuniform source and aberration lens, (a) linewidth at $2 \mu \mathrm{m}$ and (b) $5 \mu \mathrm{m}$.

a uniform intensity distribution but it also provides different intensity distributions to optimize the feature of the wafer, through transfer lens and lithography lens.

\subsection{Programming UVLED Array}

The uniform source is essential for producing the smallest linewidth in a printed integrated circuit upon substrate through mask. The lens, which collimating and aligning all rays in one aperture (CAAA), is delivering the UVLED array with transfer optics, to provide uniform or variable distribution by programming each UVLED to fit the aligner or stepper. The uniformity of the average intensity has demonstrated an excellent ability to fit for high-quality lithography either in aligner exposure or with steppers while all UVLEDs in the array are switched on for field uniform intensity distribution.

Moreover, while the defected feature of the wafer is formed due to the aberration of the lithographic lens optics, the programming UVLED array with calculated distribution delivered through CAAA lens may compensate it.

\section{Design and Fabrication of Transfer Lens}

\subsection{Theory for Amending the Defect Feature in Wafer}

While the source is uniform, the defect features in the wafer are produced by an aberration of the lens convoluted with the mask pattern feature. If the aberration is exposed, defected wafer can be quantized by a Fourier transform lens to find intensity distribution of whole field within the tolerance; thus, adjusting the wavefront error comes from the uneven intensity in the wafer to convert them to the location and intensity of individual UVLED. The UVLED array passing through the transfer lens to the mask and then delivered by the lithographic lens to the wafer forms the expected feature. Transfer lens is designed as below.

First, the UVLED array, integrated with many UVLEDs and installed in a plane, is arranged in a 2-D matrix form. Assume each ULVED as a point source radiated with an angular distribution angle of the $k$ wave vector, and the lens is used to transfer the UVLED array to collimate each wave vector direction at the stop position.

The wavefunction in the aperture of the emitting source as single UVLED is static and expressed as

$$
\mathrm{U}(x, y, z)=U_{0} \frac{e^{i \mathbf{k r}}}{r}=U_{0} \frac{e^{i\left(\mathbf{k}_{\mathbf{x}} \mathbf{x}+\mathbf{k}_{\mathbf{y}} \mathbf{y}+\mathbf{k}_{\mathbf{z}} \mathbf{z}\right)}}{\sqrt{x^{2}+y^{2}+z^{2}}},
$$

where $k$ is the wave vector and $r$ is the distance between the UVLED and the stop position, in which the mask is placed. The UVLED emitted ray has its divergent angle as the $k$ vector can be 


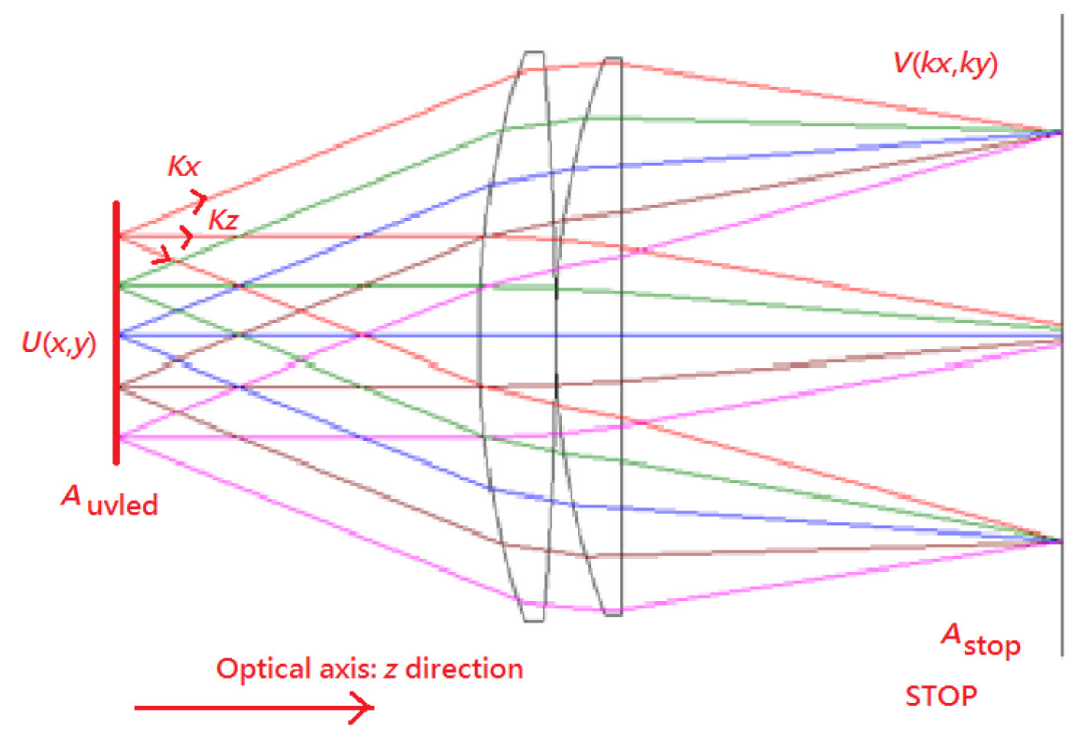

Fig. 2 The transfer lens for UVLED array.

expressed as field angle, $\mathbf{k}_{\mathbf{x}}$ from $\mathbf{k}_{\mathbf{x} \text { max }}$ to $\mathbf{k}_{\mathbf{x} \min }, \mathbf{k}_{\mathbf{y}}$ from $\mathbf{k}_{\mathbf{y} \max }$ to $\mathbf{k}_{\mathbf{y} \min }$, due to the arrangement of UVLED array telecentric, as $\mathbf{k}_{\mathbf{z}}$ is along the optical axis direction, expressed as in Fig. 2.

The UVLED array distributes rays in an area $A_{\text {UVLED }}$. The light of the UVLED array that propagates through the transfer lens will be transferred to the stop position, and each wave vector will be in the order of angles $\mathbf{k}_{\mathbf{x}}$ and $\mathbf{k}_{\mathbf{y}}$, collimated propagation; thus, the light will be distributed in the clear aperture $A_{\text {stop }}$ area as a function $\mathbf{K}$ space. ${ }^{2-5}$

By assuming the continuous distribution of UVLED along the $x$ and $y$ axes, the total transmittance $U$ of the UVLED array at the entrance pupil in the $z$ direction can be expressed as

$$
\mathrm{U}=\iint U(x, y) \mathrm{d} x \mathrm{~d} y=\iiint^{A_{\mathrm{UVLED}}} U \frac{e^{i\left(\mathbf{k}_{\mathbf{x}} \mathbf{x}+\mathbf{k}_{\mathbf{y}} \mathbf{y}+\mathbf{k}_{\mathbf{z}} \mathbf{z}\right)}}{\sqrt{x^{2}+y^{2}+z^{2}}} \mathrm{~d} x \mathrm{~d} y .
$$

The transformation equation $V$ is the total intensity distribution in the UVLED at the stop position. By multiplying $U$ with the transfer function $H(x, y, z)$, it becomes

$$
\begin{aligned}
\mathrm{V} & =H U=\iint^{A \text { stop }} V\left(\mathbf{k}_{\mathbf{x}}, \mathbf{k}_{\mathbf{y}}\right) \mathrm{d} k_{x} \mathrm{~d} k_{y} \\
& =\iiint^{A s t o p} H(x, y, z)\left(\iint^{A_{\text {UVLED }}} U \frac{e^{i\left(\mathbf{k}_{\mathbf{x}} \mathbf{x}+\mathbf{k}_{\mathbf{y}} \mathbf{y}+\mathbf{k}_{\mathbf{z}} \mathbf{z}\right)}}{\sqrt{x^{2}+y^{2}+z^{2}}} \mathrm{~d} x \mathrm{~d} y\right) \mathrm{d} k_{x} k_{y} \\
& =\iint \sqrt{x^{2}+y^{2}+z^{2}}\left(\iint^{A_{\text {UVLED }}} U \frac{e^{i\left(\mathbf{k}_{\mathbf{x}} \mathbf{x}+\mathbf{k}_{\mathbf{y}} \mathbf{y}+\mathbf{k}_{\mathbf{z}} \mathbf{z}\right)}}{\sqrt{x^{2}+y^{2}+z^{2}}} \mathrm{~d} x \mathrm{~d} y\right) \mathrm{d} k_{x} k_{y} \\
& =\iint^{A \text { stop }} \mathrm{U} e^{i\left(\mathbf{k}_{\mathbf{x}} \mathbf{x}+\mathbf{k}_{\mathbf{y}} \mathbf{y}+\mathbf{k}_{\mathbf{z}} \mathbf{z}\right)} \mathrm{d} k_{x} k_{y},
\end{aligned}
$$

here the transfer function is defined as $\mathrm{H}(\mathrm{x}, \mathrm{y}, \mathrm{z})=\sqrt{x^{2}+y^{2}+z^{2}}$ by considering the lensguiding light aiming at the aperture position to collimate the wave vectors $\mathbf{k}_{\mathbf{x}}$ and $\mathbf{k}_{\mathbf{y}}$ at the stop position in the $\mathrm{z}$ direction, $\mathrm{U}$ is the total intensity distribution at UVLED array, and $\mathrm{V}$ is the total intensity distribution in the UVLED light source at the stop position.

$$
\begin{gathered}
\mathrm{V}=\mathrm{HU}, \\
\mathrm{U}=H^{-1} \mathrm{~V},
\end{gathered}
$$


here $\mathrm{H}$ is a transfer function as CAAA lens to transfer rays of $\mathrm{U}$ to $\mathrm{V}$, thus $\mathrm{V}$ also can transfer to $\mathrm{U}$ through $\mathrm{H}^{-1}$.

For the defect images caused by aberrations, the point-spread function (PSF) will exhibit broad spreading, thus introducing defect in the image. In an ideal condition, the PSF is a distribution of the sinc function, and by the Fourier transforms of the wavefront of the lithographic lens, each point of the image is simulated by the object times the PSF ${ }^{5-8}$

The defected feature of the wafer can be amended by calculating the deviation error between exposed feature in the wafer and the designed feature as a function $V$ in the stop position, which is mask placed. By the Fourier reverse transfer function [Eq. (5)], the function $U$ can be solved to program various distributions by programming each single UVLED position and intensity in the array. Thus, the corrected feature can be obtained by programming the UVLED array with a transfer lens.

In this study, CODE V, optical design program ${ }^{9}$ was used to design the illumination lens, and LighTools program has been applied in the calculation of the illumination to provide sufficient information for analysis. ${ }^{10}$

\subsection{Optical Design}

A transfer lens was designed by collimating all the lights from the UVLEDs in the array using the newly proposed aperture method - collimating and aligning all rays in aperture (CAAA) method. The lens was designed and all the wavefronts of light were summed together at the aperture and the propagation rays were collimated in each specific wave vector direction, as shown in Fig. 2.

Based on the specification, the illumination design of the lens is such that it guides all the rays from each UVLED in the 2-D array to converge together at the aperture; thus, each field at aperture is emitted in a telecentric manner. Each individual emitting UVLED as point light source emitted light to the stop through lens to become a collimated light as plane wave. ${ }^{11}$ Based on the specification, the illumination design arranges all the lens to guide all the rays that converge together at the aperture; thus, each field at the aperture is emitted in a telecentric manner. In Fig. 3, the lens is composed of four lenses, $F / \#=1.4$, and the magnification is 2. This design is a one side conjugate with telecentric sources, and all the marginal rays from each UVLED are collimated for each emitting field, forming a uniform and coherent illumination. In addition, wavefront of each emitting UVLED are summed together at stop, which is a place put mask, in uniform intensity distribution for aligner or variable intensity distribution for wafer defected compensation of steppers.

\subsection{Uniform Source}

The optical design and fabrication of the lithographic source is for a multiple-purpose aligner to eliminate the fatal feature caused by a nonuniform source. The illumination design requirement was implemented to transfer from the wide divergent angle and nonuniform UVLED source to the smallest divergent angle and uniform source, respectively. ${ }^{11-15}$

The optical design for the UVLED array is to design transfer lens to take the UVLED array as an input to increase the emitting volume and decrease the divergent angle. According to the specification, the size of the UVLED array is $150 \mathrm{~mm} \times 150 \mathrm{~mm}$. The half-angle divergence is

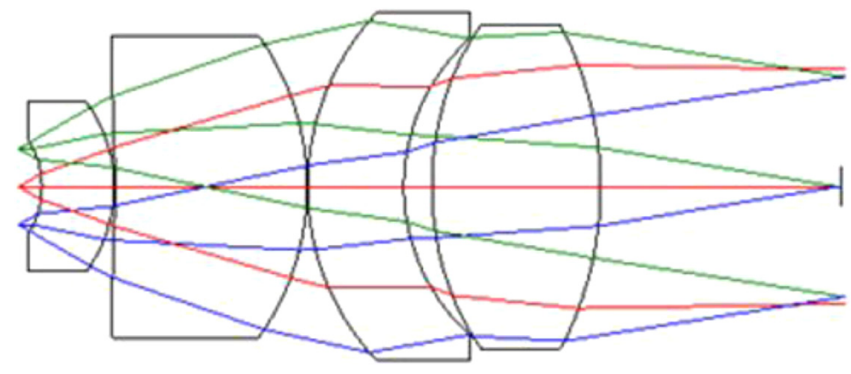

Fig. 3 Four lenses forming the transfer lens. 


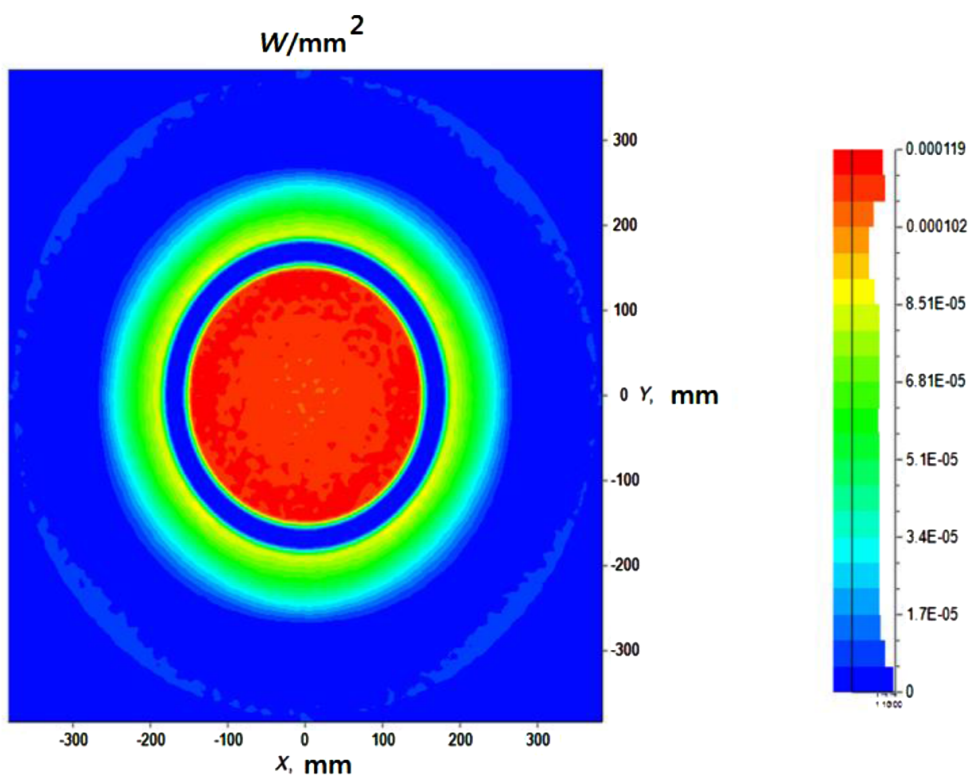

Fig. 4 The UVLED array light source near the field intensity distribution.

$60 \mathrm{deg}$ of each UVLED. Designing is performed by considering the light source as the telecentric object and using the collimating lens to form a collimated aperture as the stop position. The output of light source is a 12-in. square, and the uniformity in 14-in. diameter is higher than 98\%, as shown in Fig. 4.

\subsection{Verification of Uniformity}

Figure 5 shows the UVLEDs in the 2-D matrix array passing through the transfer lens. All UVLEDs are off in Fig. 5(a), all lights on are shown in Fig. 5(b). Due to lens set $F / \#$ from 80 to 120 , good to be a source, it is allowed to the extended depth of field to fit for alignment of the mask. All wavefront at stop are summed together at the aperture to produce the summation of all UVLEDs, thus averaging all direction of the energy from all UVLEDs. Figure 6 shows the intensity distribution of a $15 \times 15$ UVLED array, as shown in Fig. 6(a), and that in the image plane through the transfer lens, as shown in Fig. 6(b).

\subsection{Fourier Transfer Lens}

A Fourier transform plays an important role in wave propagation in homogeneous media and in the treatment of wave propagation through lenses. The amplitude distributions in the front and back focal planes of a lens form a good approximation. In a Fourier pair, the PSF is the Fourier

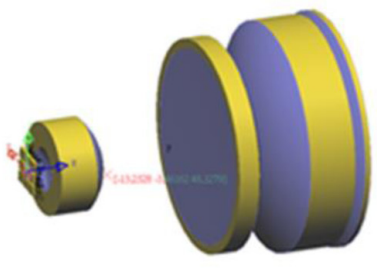

(a)

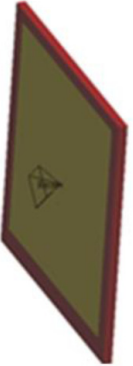

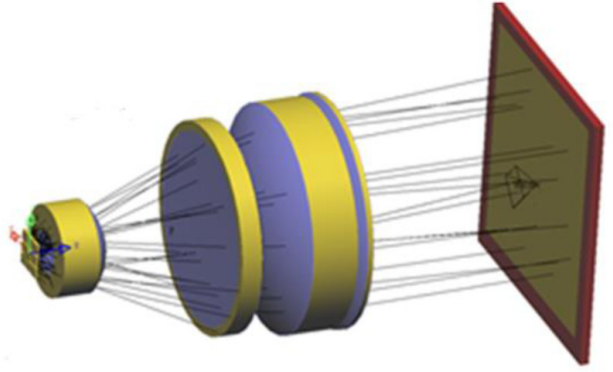

(b)

Fig. 5 UVLEDs in the 2-D matrix array passing through the transfer lens: (a) light off and (b) light on. 
$W / \mathrm{mm} 2$

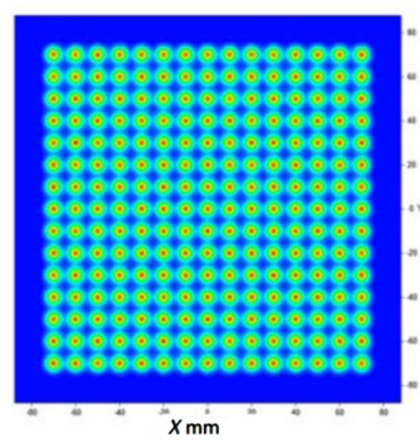

(a)

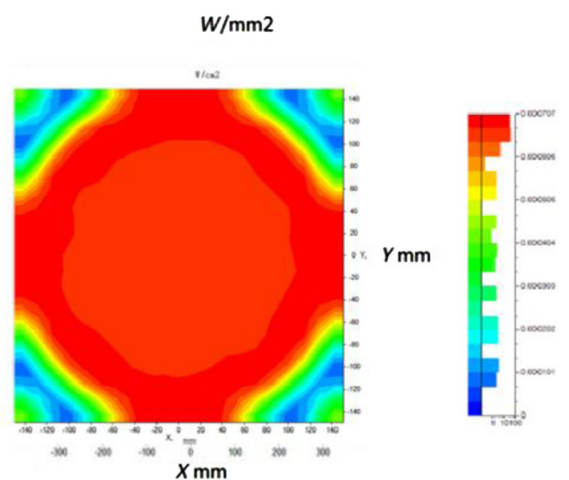

(b)

Fig. 6 (a) Intensity distribution close to $15 \times 15$ UVLED array and (b) intensity distribution in the wafer plane through the transfer lens.
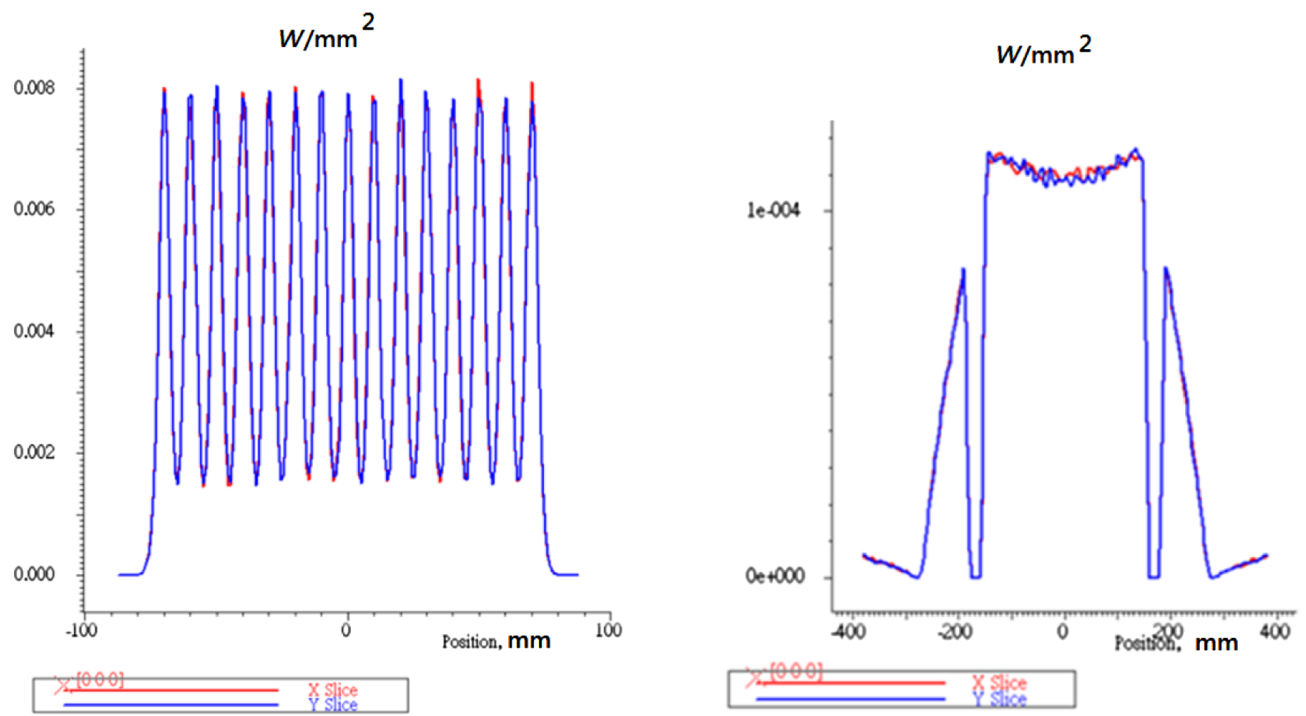

Fig. 7 (a) A 1-D intensity distribution of input array and (b) 1-D intensity distribution after light passes through the transfer lens.

transform of the complex amplitude distribution in the exit pupil. ${ }^{8}$ Here, the lens also demonstrated Fourier transform of the UVLED array as an aperture to the PSF, as shown in Fig. 7. The lens exhibits the Fourier transform function, and every PSF is overlapped by all the fields. Figure 7(a) shows one-dimensional (1-D) intensity distribution of input array, and Fig. 7(b) shows 1-D intensity distribution after light passes through the transfer lens.

\section{Illumination by Programming the UVLED Array}

\subsection{Programming Source}

The UVLEDs array with a matrix of $15 \times 15$ elements formed as an object to emit light passes through the lens and distributes light to an aperture to form different distribution light fields. Figures 8 and 9 show the light distribution from the UVLED array, respectively, with a cross mark and circle mark passing through the transfer lens. Figure 8(a) shows the intensity distribution of UVLED array with the cross mark, and Fig. 8(b) shows the intensity distribution of UVLED after passing through transfer lens. Figure 9(a) shows the intensity distribution of UVLED array with the circle mark, and Fig. 9(b) shows the intensity distribution after passing through the transfer lens. 


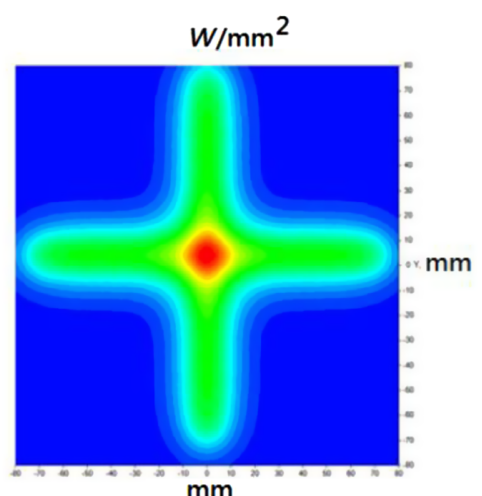

(a)

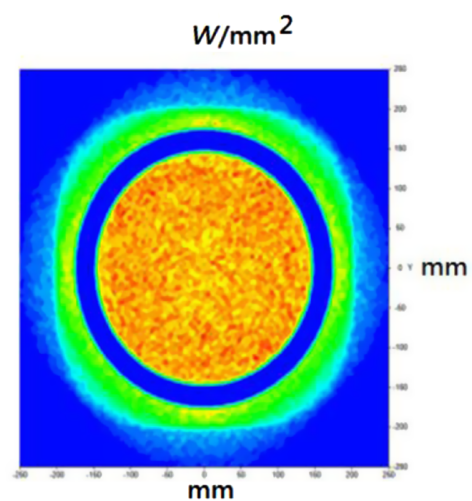

(b)

Fig. 8 (a) Intensity distribution of UVLED array with the cross mark and (b) passing through transfer lens.

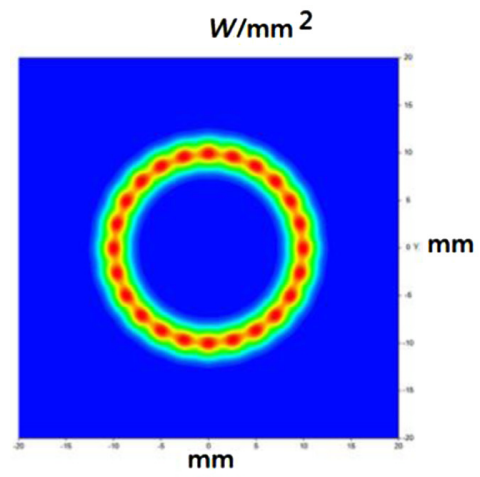

(a)

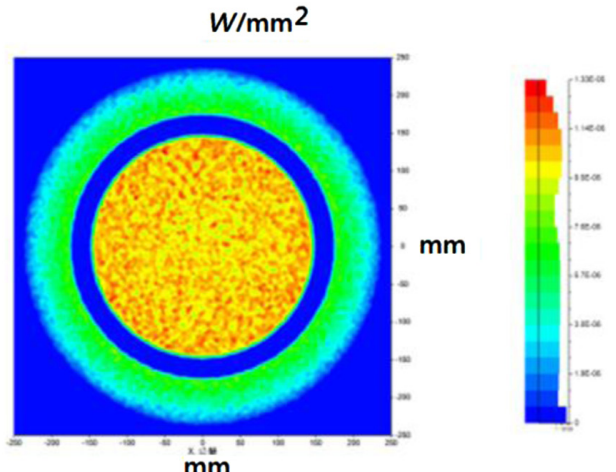

(b)

Fig. 9 Intensity distribution of UVLED array (a) with the circle mark and (b) after passing through the transfer lens.

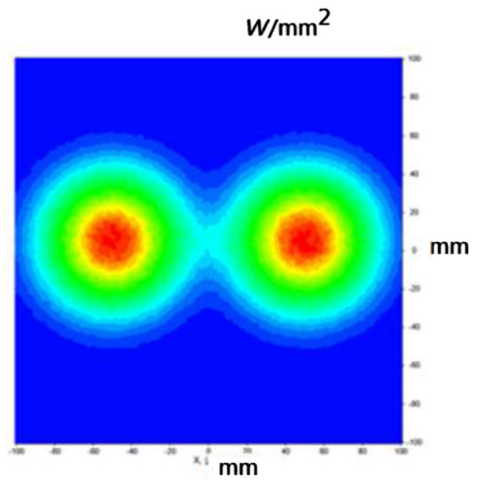

(a)

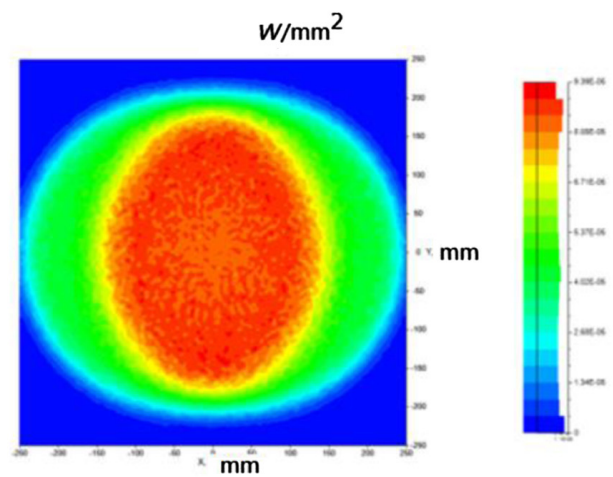

(b)

Fig. 10 Intensity distribution of UVLED array (a) with dipole source and (b) through the transfer lens at the stop position.

\subsection{Programmable Source}

The light source can provide the aberration distribution by taking two sources as dipole and four sources as the quadrupole to form comma and astigmatism distribution. Figures 10 and 11 show 


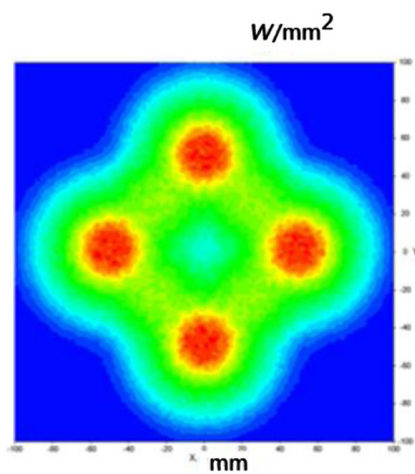

(a)

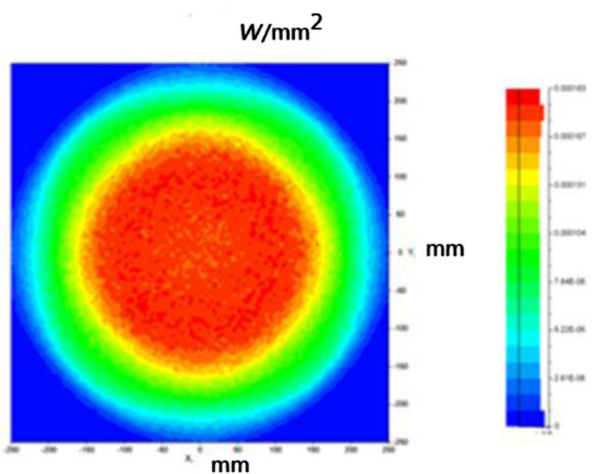

(b)

Fig. 11 The intensity distribution of UVLED array with a (a) light source as the quadrupole and (b) passing through the transfer lens at the stop position.

the intensity distributions for the dipole and quadrupole sources of the UVLED array through the transfer lens, respectively.

We used the UVLED array as a 2-D plane emitted from a programmable light source. Each UVLED could be adjusted in intensity, divergent angle, and light field to form a programmable light intensity source, uniform distribution, or nonuniform demanded distribution.

\section{Results and Analysis}

Figure 12 shows the experiment setup for an aligner, which is composed of UVLED array, transfer lens, and stop platform. The UVLED array is $N \times N$ UVLED arrays with programmable time

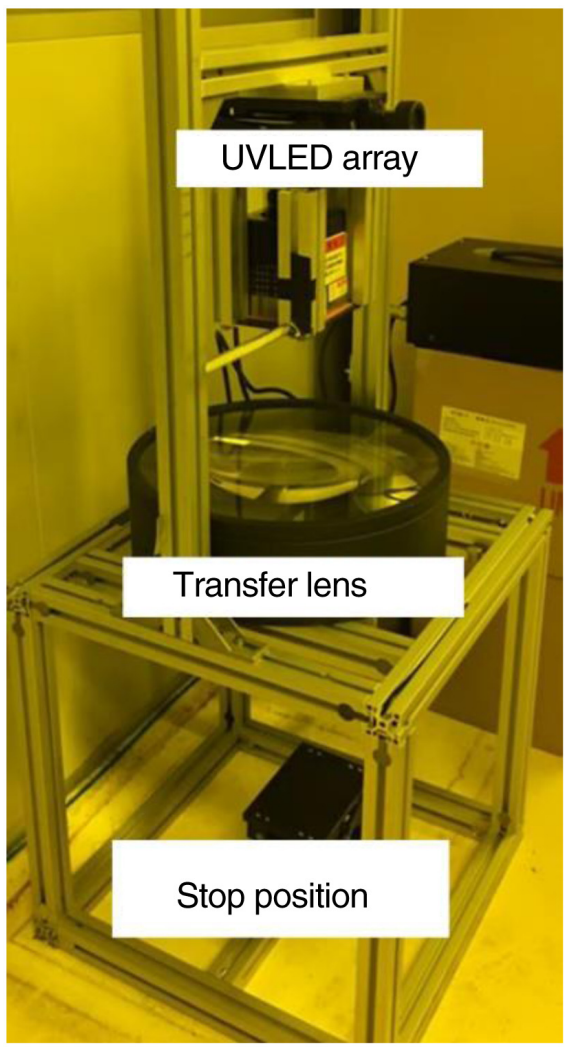

Fig. 12 UVLED array aligner composed of UVLED array, transfer lens, and stop platform. 


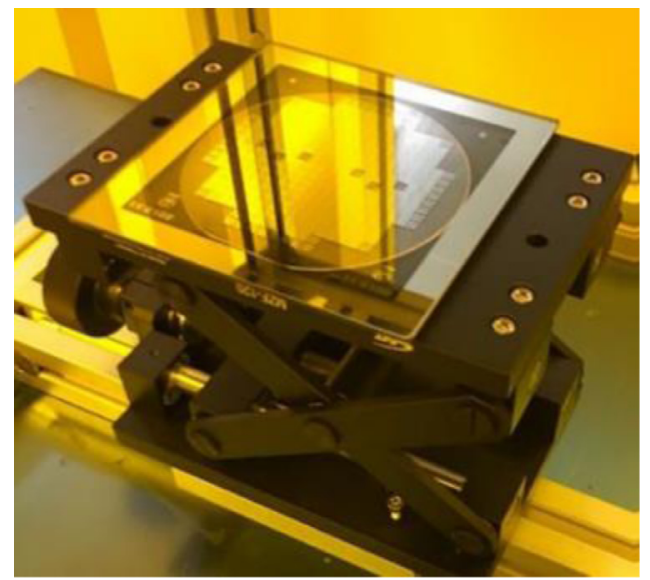

Fig. 13 Stop platform is distance adjustable and is able to make to wafer for soft contact and proximity

and lamination setting controller. The transfer lens is fabricated according to the specification, a 14-in. transfer lens. Stop platform is distance between wafer and mask adjustable, thus, it can make to wafer for soft contact and proximity, as shown in Fig. 13. Figure 14 shows the power intensity distribution in the $14 \mathrm{in}$. wafer position to produce uniform light field by taking power for each different location with scale within $10 \mathrm{~mm}$.

The results obtained using the programming UVLED array sources to make the smallest linewidths in the wafer are described as follows.

\subsection{Soft Contact and Proximity Mode}

Figures 15(a) and 15(b) show the contact mode, 90- versus 5- $\mu$ m duty-cycle mask to form features in silica wafer, and 5- $\mu \mathrm{m}$ feature is clear, and whole field is even and smooth, compared with Fig. 1 with a significant improvement. The proximity mode (the distance between the mask and wafer is larger than $700 \mu \mathrm{m}$ ) is also carried out.

By programming the UVLED array source through the transfer lens, the contact mode achieved a small light pitch at $2 \mu \mathrm{m}$ with high uniformity in Figs. 16(a) 2, 16(b) 3, 16(c) 4,

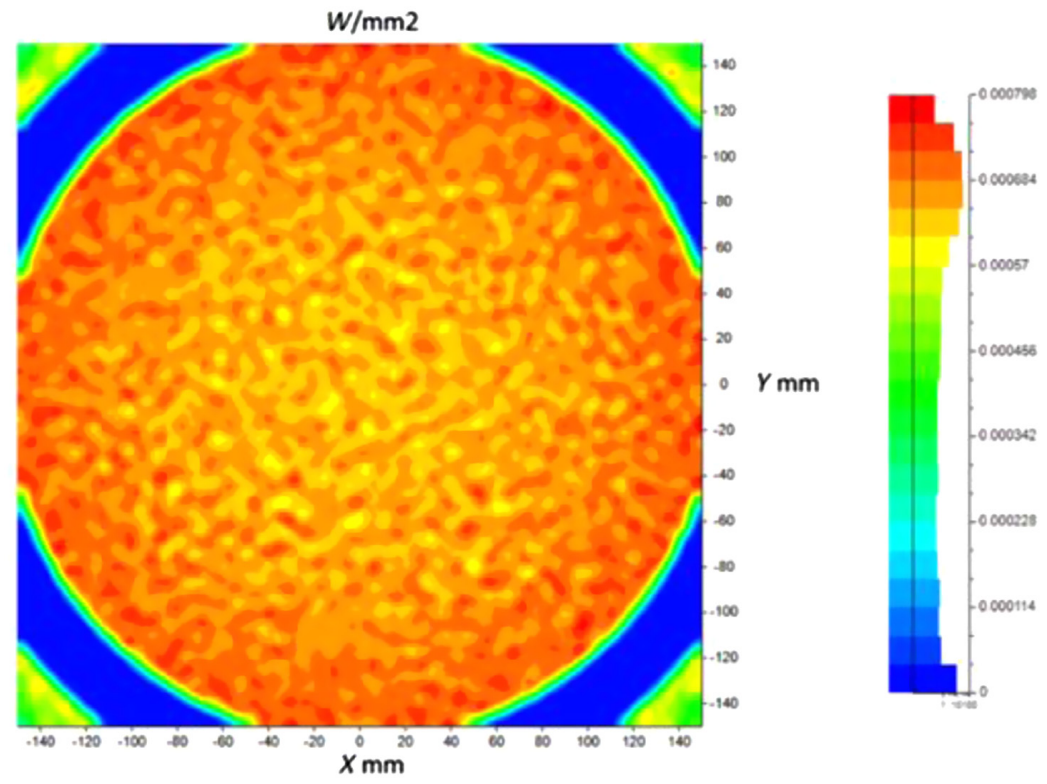

Fig. 14 The power intensity distribution in the 14-in. wafer position to produce uniform light field. 


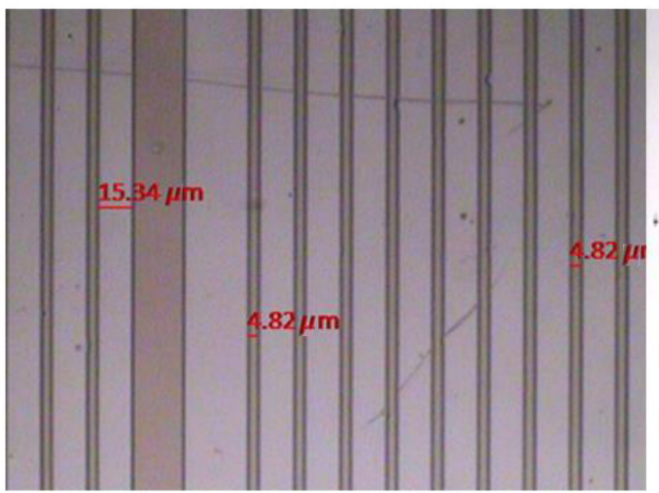

(a)

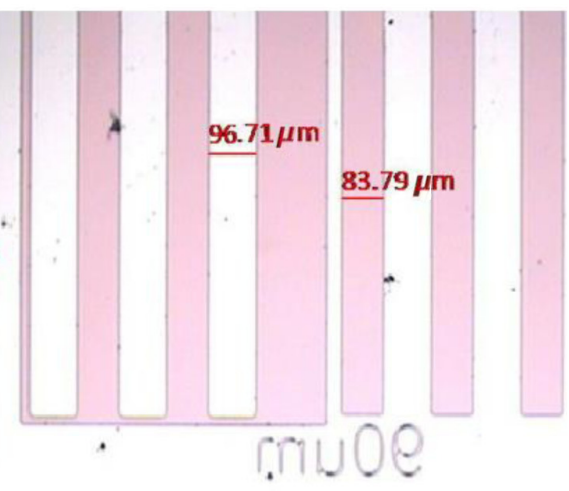

(b)

Fig. 15 (a) The 5- $\mu \mathrm{m}$ versus (b) $90-\mu \mathrm{m}$ duty-cycle mask to form features in silica wafer.

16(d) 5, 16(e) 6, and 16(f) $7 \mu \mathrm{m}$. Figure 17 shows CD bias for different linewidths from 2 to $10 \mu \mathrm{m}$, which is $<1 \mu \mathrm{m}$ constantly. Furthermore, the small light pitch can be achieved below $1 \mu \mathrm{m}$ by fine adjustment of the UVLED array distances and mask/wafer position. Figure 18 shows high-performance aligner, and it can perform a quality feature.

\subsection{Stepper}

One of applications for noncontact lithography platform, shown in Fig. 19, is by programming the UVLED array emitted through the transfer lens, then passing through a mask, and finally, the lens formed the IC pattern in the platform's wafer with $5 \%$ error in demanded size features.

(a)

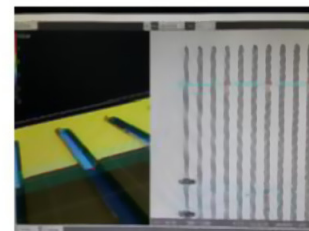

(b)
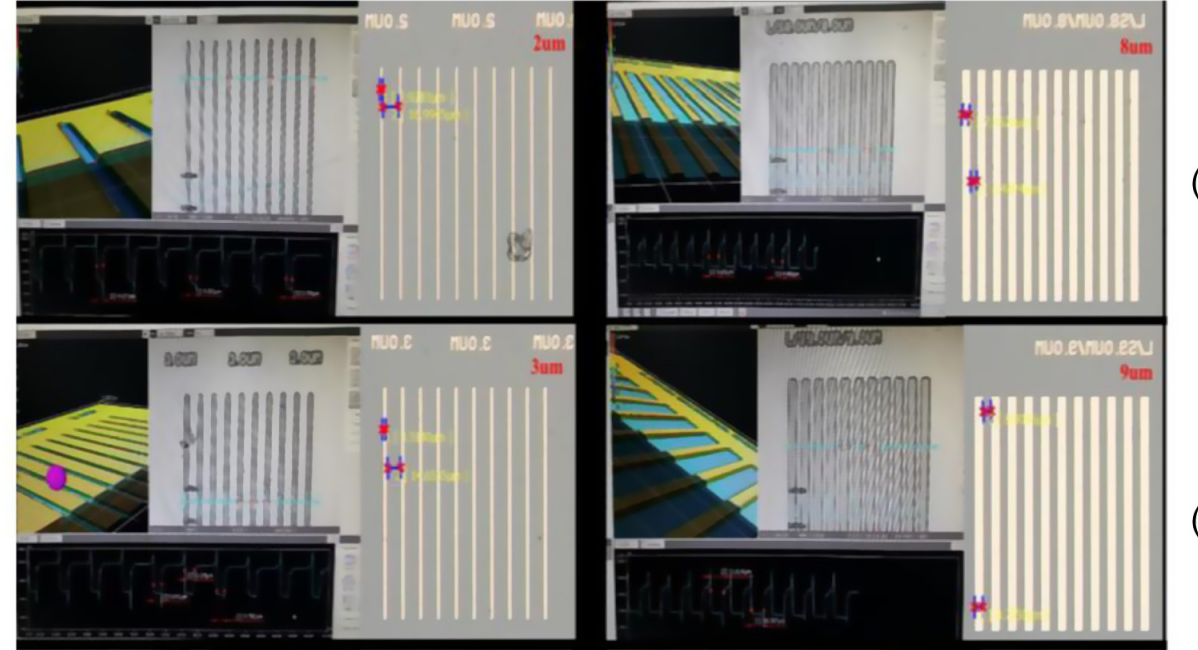

(c)
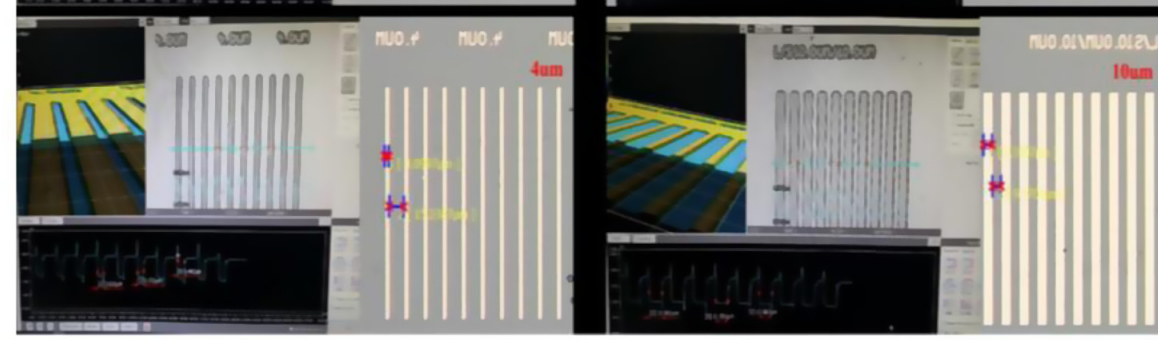

(d)

(e)

(f)

Fig. 16 Wafer features taken from Keyence laser microscope: (a) 2, (b) 3, (c) 4, (d) 5, (e) 6, and (f) $7 \mu \mathrm{m}$. 
Huang: Programmable ultraviolet light-emitting diode array with a transfer lens...

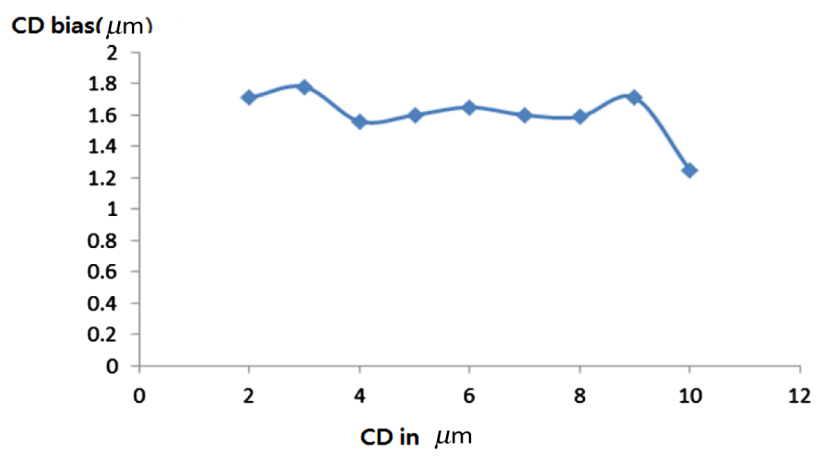

Fig. $17 \mathrm{CD}$ bias for different linewidths from 2 to $10 \mu \mathrm{m}$.

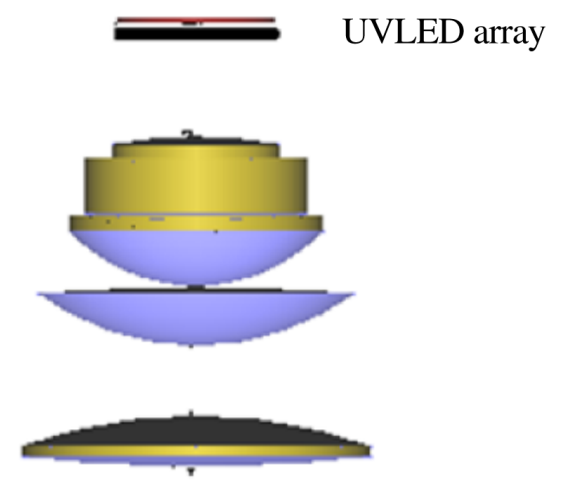

Mask

Wafer

Fig. 18 High-performance aligner.

Figure 20 shows the circuit defected exposure and fixed by the UVLED array programmable source. Figure 20(a) shows the original pattern. Figure 20(b) shows the defect pattern through the lens and Fig. 20(c) shows the corrected pattern by applying UVLED programming. Coma lens aberration, as in Fig. 10, is induced by programming the UVLED array with the transfer lens, which can be compensated by tuning the intensity distribution in UVLED and a limit diameter as aperture of source, to amend aberration and to form the qualified feature in wafer.

The transfer lens demonstrates the Fourier transfer function and it can transfer the UVLED array to the desired distribution pattern, not only uniform distribution but also variable distribution for lithographic usage. The difference between two optics-current work and Koehler illumination is that the CAAA lens aligns all rays from each UVLED are summed together at a common aperture for coherent or noncoherent uniform or demanded distribution, whereas the Koehler illumination imaged the source into the lithographic lens and only offers the uniform distribution at mask position. 

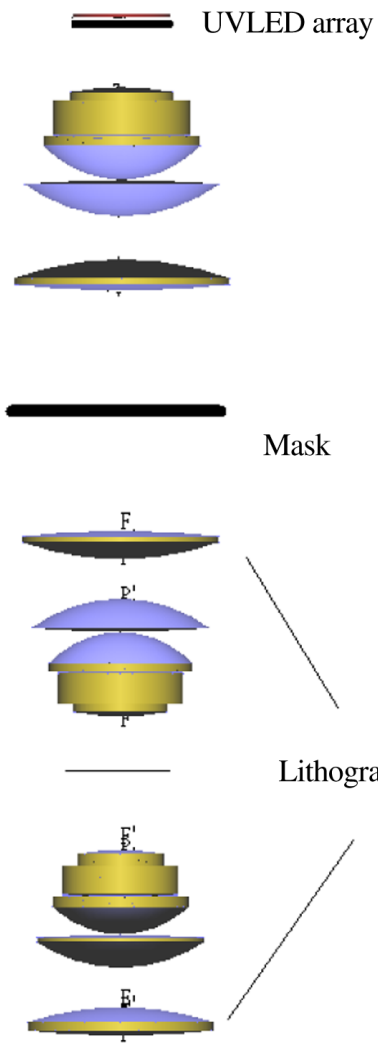

Lithographic lens

Wafer

Fig. 19 UVLED array stepper.

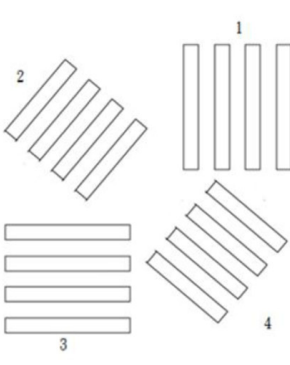

(a)

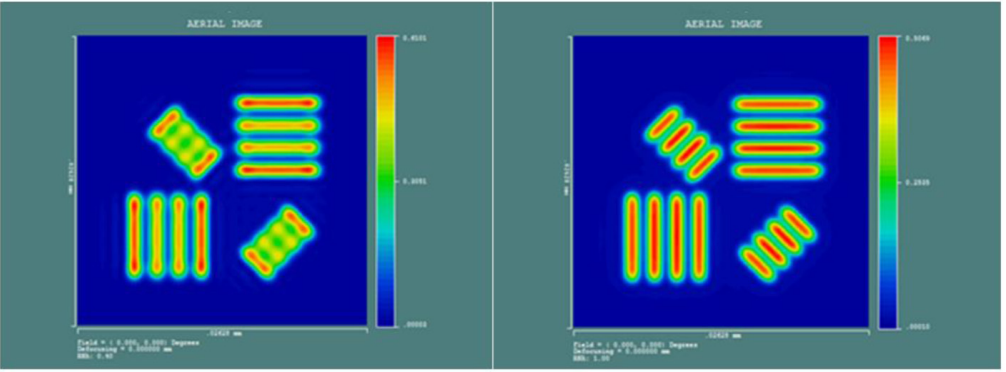

(b) (c)

Fig. 20 The circuit with quadruple patterns by UVLED programmable source: (a) the original pattern on mask, (b) defected pattern, and (c) corrected pattern.

\section{Conclusion}

The work presented a programmable UVLED array with adjustable light source collimated optics as the transfer lens. The source shows that the printed wafer was improved. In addition, a programmable UVLED array with collimated optics acts as a transfer lens for uniform intensity distribution to mask, acting as the Fourier transfer lens. Moreover, to compensate for the incomplete printed feature and possible use in other advanced sources, the programmable UVLED array source has also shown to be a good substitute for metal-formed quadrupole and dipole apertures. 


\section{Acknowledgments}

This work was supported by a grant from the Ministry of Science and Technology, Taiwan, Republic of China (Grant Nos. 107-2622-E-492-004-CC3 and 108-2622-E-492-014-CC3). The support from M\&R Optic Electronic Co. and Control Optics Taiwan Co. are also deeply appreciated.

\section{References}

1. K. Wong, Optical Imaging Projection in Projection Microlithography, SPIE Press, Bellingham, Washington (2005).

2. J. Huang, "Optical design of a 1-to-1 lithography projection," Opt. Rev. 23(5), 870-877 (2016).

3. J. Huang, "The ultra-violet partial coherence modulation transfer function for lithography," Proc. SPIE 10147, 101471L (2017).

4. H. H. Hopkins, "On the diffraction theory of optical images," Proc. R. Soc. Lond. Ser. A (1953).

5. J. W. Goodman, Introduction to Fourier Optics, McGraw-Hill, New York (1968).

6. F. Zernike, "Phase difference microscopy," Z. Tech. Phys. 16, 454 (1935).

7. G. R. Fowles, Introduction to Modern Optics, Rinehart and Winston, Inc., Holt (1972).

8. B. E. A. Saleh and M. C. Teich, Fundamentals of Photonics, Wiley Interscience, Hoboken, New Jersey (1991).

9. Code V 12.4, Synopsis Inc., USA (2020).

10. LighTools 9.0, Synopsis Inc., USA (2020).

11. S. Ookubo, Riken, Saitama University Co-develop UV-LED with $227.5 \mathrm{~nm}$ Emission Wavelength, Nikkei Electronics (2007).

12. F. Y. Ciou et al., "Investigation of uniformity field generated from freeform lens with UV LED exposure system," Proc. SPIE 9383, 93830S (2015).

13. S. Suzuki and Y. Matsumoto, "Lithography with UV-LED array for curved surface structure," Microsyst. Technol. 14, 1291-1297 (2008).

14. A. V. Arecchi, T. Messadi, and R. J. Koshel, Field Guide to Illumination, SPIE Press, Bellingham, Washington (2007).

15. C.-C. Su et al., "Collimating lamp with well color mixing of red/green/blue LEDs," Opt. Express 20(51), A74-A84 (2012).

Jiun-Woei Huang is a chief scientist of the National Innovation Project for near-eye augmented reality glass and an adjunct professor, the Institute of Applied Mechanics, National Taiwan University. He received his BS and MS degrees in physics from National Taiwan Normal University in 1977 and 1981, respectively, and his PhD in physics from Texas Christian University, USA. He is the author of more than 20 patents in the USA and Taiwan, and a book The Design and Fabrication of Optro-Mechtronic System. His current research interests include optical design and fabrication of lithography, spectroscopy, AR and VR, and display optics. He is a member of SPIE, OSA, and a life member of Sigma Xi. 